Article

\title{
Facile Control of the Porous Structure of Larch-Derived Mesoporous Carbons via Self-Assembly for Supercapacitors
}

\author{
Xin Zhao ${ }^{1,2, *}$, Wei Li ${ }^{2}$, Honglei Chen ${ }^{1}$, Shoujuan Wang ${ }^{1}$, Fangong Kong ${ }^{1}$ and Shouxin Liu ${ }^{2, *}$ \\ 1 Key Lab of Pulp and Paper Science and Technology of Ministry of Education (Shandong Province), \\ Qilu University of Technology, Jinan 250353, China; shaming007@163.com (H.C.); \\ nancy5921@163.com (S.W.); kfgwsj1566@163.com (F.K.) \\ 2 College of Material Science and Engineering, Northeast Forestry University, Harbin 150040, China; \\ liwei19820927@126.com \\ * Corresponding author: zhaoxin_zixi@126.com (X.Z.); liushouxin@126.com (S.L.); \\ Tel.: +86-0531-89631681 (X.Z.); +86-0451-82191204 (S.L.)
}

Received: 26 October 2017; Accepted: 17 November 2017; Published: 20 November 2017

\begin{abstract}
Mesoporous carbons have been successfully synthesized via self-assembly using larch-based resins as precursors and triblock copolymers as soft templates. The porous structure of mesoporous carbons can be tailored by adjusting the ratio of hydrophilic/hydrophobic (EO/PO) units owing to interfacial curvature. Interestingly, the porous structures show a distinct change from vortex-like to worm-like pores, to stripe-like pores, and to ordered two-dimensional hexagonal pores as the ratio of hydrophilic/hydrophobic units increases, indicating the significant effect of $\mathrm{EO} / \mathrm{PO}$ ratio on the porous structure. The mesoporous carbons as supercapacitor electrodes exhibit superior electrochemical capacitive performance and a high degree of reversibility after 2000 cycles for supercapacitors due to the well-defined mesoporosity of the carbon materials. Meanwhile, the superior carbon has a high specific capacitance of $107 \mathrm{~F} \cdot \mathrm{g}^{-1}$ in $6 \mathrm{M} \mathrm{KOH}$ at a current density of $10 \mathrm{~A} \cdot \mathrm{g}^{-1}$.
\end{abstract}

Keywords: larch; mesoporous carbons; soft template; EO/PO ratio; supercapacitors

\section{Introduction}

Due to the increasing sustainable and energy concerns caused by excessive consumption of fossil fuels, energy storage devices, such as fuel cells, lithium-ion batteries, and supercapacitors, are considered alternative candidates for practical applications. Supercapacitors, as promising new-type electrochemical devices with high power density, excellent cyclic performance, and less sustainable development pollution, have attracted considerable attention [1-5]. Generally, supercapacitors can be divided into electrical double-layer capacitors (EDLCs) and pseudocapacitors according to their charge storage mechanism. EDLCs possess an electrostatic attraction at the interface of electrodes and electrolytes with charge accumulation; however, pseudocapacitors exhibit faradic redox reactions [6,7]. The development of supercapacitors is very useful for the large-scale applications of portable electronic systems and automotives due to their high-power density, excellent reversibility, and long cycle life.

Recently, mesoporous carbons have received considerable attention for the development of high-performance supercapacitors due to their high surface area, uniform and tunable porous structure, and chemical inertness [8-10]. These unique characteristics make them ideal candidates for supercapacitor electrodes with high power density and energy density. Furthermore, their uniform mesopores facilitate the transportation of electrolyte ions, resulting in better electrochemical performance at high current densities [11-13]. Indeed, the mesoporous carbons exhibit significant 
advantage over the microporous activated carbons under short duration and discharge or recharge [14]. Therefore, the development of ordered mesoporous carbons with high porosity for the supercapacitors is an important issue.

The ordered mesoporous carbons are usually prepared with a hard template method; however, this method is complex, time-consuming, and inefficient, such that it is unsuitable for industrial applications. Recently, the synthesis of ordered mesoporous carbons with the soft template method has attracted increasing attention because it is a facile, time-saving, and efficient strategy. The synthesis technology is based on the self-assembly of copolymer molecules with carbon precursors via hydrogen bond $[15,16]$. The porous structure of mesoporous carbons prepared with the soft template method can be tuned by the type of solvent, block strength, and carbonization temperature $[17,18]$. Generally, phenol resins are the main carbon resources for the synthesis of mesoporous carbons via soft template. However, biomasses as renewable resources have limited report for the preparation of mesoporous carbons [19-21]. Our group previously reported the synthesis of different morphologies of mesoporous carbons (e.g., carbon films, carbon spheres, and carbon foam) with controllable porous structures using liquefied wood as carbon resources [22-24]. Meanwhile, we used triblock copolymer Pluronic F127 and P123 as soft templates for the preparation of the mesoporous carbons with 2-D hexagonal and spherical ordered channel structures via the direct carbonization of organic-organic self-assembled composites [25]. The carbon precursors with abundant hydroxyl groups can strongly interact with the polar parts of structure-directing block-copolymers, leading to the self-assemble of precursors around micelles. These results demonstrated that the wood can be utilized to synthesize the mesoporous carbons with high porosity for supercapacitors.

Herein, we present a facile synthesis of mesoporous carbons with different porous structures (ordered and disordered) via organic-organic self-assembly using liquefied larch as carbon resources and mixture copolymers as templates. The porous structure of the carbons is further tailored by the ratio of hydrophilic/hydrophobic units (EO/PO). Furthermore, we illustrate the mechanism of the self-assembly and the interaction between larch-based resins and templates, in an attempt to attract more attention and determine the real value of these mesoporous carbons. Furthermore, these mesoporous carbons as supercapacitor electrodes are tested to evaluate the capacitive performance, and the effect of the morphology and porous structure of carbons on the electrochemical properties is investigated.

\section{Experimental}

\subsection{Preparation of Ordered Larch-Based Mesoporous Carbons}

The synthesis of the liquefied larch was based on our previous work [25]. In a typical synthesis, larch sawdust (10 g), phenol (30 mL), sulfuric acid $(98 \%, 1 \mathrm{~mL})$, and phosphoric acid $(85 \%, 2 \mathrm{~mL})$ were placed into a three-necked glass. The mixture was heated under reflux at $120{ }^{\circ} \mathrm{C}$ for $1 \mathrm{~h}$. Then the mixture was filtered with methanol, and then adjusted to neutral using sodium hydroxide, followed by the filtering of the resulting precipitate. The filtrate was concentrated by vacuum distillation at $40{ }^{\circ} \mathrm{C}$, and liquefied larch was obtained.

The F127 and P123 are expressed with poly(ethylene oxide)-poly(propylene oxide)-poly (ethylene oxide), denoted as $\mathrm{EO}_{m}-\mathrm{PO}_{n}-\mathrm{EO}_{m}$ in which the $m_{1}$ and $n_{1}$ of $\mathrm{F} 127$ is 106 and 70 and the $m_{2}$ and $n_{2}$ of P123 is 20 and 79, respectively. The EO groups can self-assemble with resins forming mesoporous structure via hydrogen bonds. The EO groups mainly contribute to the pore size of the carbons. For the synthesis of the mesoporous carbons, a mixture of $x \mathrm{~g}(0,2,4.5,5.5,6,8$, and $10 \mathrm{~g}) \mathrm{F} 127$ and $(10-x) \mathrm{g}$ P123 as the soft template was dissolved in $20 \mathrm{~mL}$ of ethanol under magnetic stirring at $30{ }^{\circ} \mathrm{C}$. In a typical synthesis, formaldehyde $(37 \%, 90 \mathrm{~mL})$ and sodium hydroxide $(3 \mathrm{~g})$ were added to the as-synthesized liquefied larch to generate larch-based resins under basic conditions. Ten-gram templates were then added and stirred at $40^{\circ} \mathrm{C}$ for $20 \mathrm{~h}$. Next, the $\mathrm{pH}$ was adjusted to 0.5 with $\mathrm{HCl}$, and the reaction was continued at $50{ }^{\circ} \mathrm{C}$ for $8 \mathrm{~h}$. The obtained mixture was dried at $80^{\circ} \mathrm{C}$ for $6 \mathrm{~h}$. 
Finally, the carbon materials were formed after carbonization under an $N_{2}$ atmosphere at $700{ }^{\circ} \mathrm{C}$ for $2 \mathrm{~h}$. The carbon materials were denoted as $\mathrm{C}-\mathrm{y}$, where $y$ is the $\mathrm{EO} / \mathrm{PO}$ ratio, calculated as follows:

$$
y=\left(x m_{1}+(10-x) m_{2}\right) /\left(x n_{1}+(10-x) n_{2}\right)
$$

where $m_{1}$ and $m_{2}$ stand for the amount of EO units of F127 and P123, and $n_{1}$ and $n_{2}$ stand for the amount of PO units of F127 and P123, respectively.

\subsection{Characterization}

The images of transmission electron microscopy (TEM) were obtained on a JEOL 2011 (JEOL, Hokkaido, Japan) apparatus operated at $200 \mathrm{kV}$. Powder X-ray diffraction patterns of CMs were measured using a Brucker D4 (Bruker, Hokkaido, Japan) powder X-ray diffractometer with $\mathrm{Cu}$ $\mathrm{K} \alpha$ radiation at $40 \mathrm{kV}$ and $40 \mathrm{~mA}$. Nitrogen sorption isotherms were measured with a Micromeritics ASAP 2020 sorptometer (Maike, Birmingham, AL, USA) using nitrogen as the adsorbate at $77 \mathrm{~K}$. All samples were degassed at $300{ }^{\circ} \mathrm{C}$ for more than $10 \mathrm{~h}$ before analysis. The surface area $\left(\mathrm{S}_{\mathrm{BET}}\right)$ was calculated using the BET method based on adsorption data in the relative pressure of $0.05-0.2$, and total pore volume was determined at the highest relative pressure. The pore size distribution (PSD) was determined via the DFT method using a cylindrical pore model with nitrogen adsorption data.

\subsection{Electrochemical Measurements}

For the fabrication of working electrodes, active materials ( $80 \mathrm{wt} \%)$, carbon black (10 wt \%), and polytetrafluoroethylene (PTFE; $10 \mathrm{wt} \%$ ) were well mixed, which were pressed onto a nickel foam that served as a current collector. The typical mass of active materials was about $10 \mathrm{mg} \cdot \mathrm{cm}^{-2}$. The electrochemical experiments were tested in a three-electrode cell, using platinum as the counter electrode, a saturated calomel electrode (SCE) ( $0.2415 \mathrm{~V}$ vs. the standard hydrogen electrode) as the reference electrode, and $6 \mathrm{M} \mathrm{KOH}$ solution as the electrolyte. Cyclic voltammetry (CV) measurements and galvanostatic charge/discharge (GCD) tests were carried out on a $\mathrm{CHI} 600 \mathrm{E}$ electrochemical workstation. The CV curves were measured at scan rates of $1-200 \mathrm{mV} \cdot \mathrm{s}^{-1}$ in a voltage range of -1 to $0 \mathrm{~V}$. The GCD curves were tested between -1 and $0 \mathrm{~V}$ at different current densities. Electrochemical impedance spectroscopy (EIS) measurements were carried out with the amplitude of $5 \mathrm{mV}$ in a frequency range of $10 \mathrm{mHz}$ to $100 \mathrm{kHz}$.

\section{Results and Discussion}

\subsection{Structural and Textural Properties of Mesoporous Carbons}

Small-angle X-ray scattering (SAXS) patterns of mesoporous carbons are shown in Figure 1. The as-synthesized CM- 0.3 shows four scattering peaks with $q$ values $(q=4 \pi \sin \theta / \lambda)$ at $0.688,0.927$, 1.208 , and $1.493 \mathrm{~nm}^{-1}$, which can correspond to the 10,11,20, and 21 reflections of two-dimensional (2-D) hexagonal mesostructure (group space, P6m) symmetry. For CM-0.5, the intensity of the four peaks has a slight reduction and shift to a low $q$-value, indicating a decrease in ordering and framework expansion. However, the scattering peaks of CM- 0.8 and CM- 1 gradually become weak with the increase in the $\mathrm{EO} / \mathrm{PO}$, which indicates the change in the ordered porous structure to the disordered structure. Moreover, for CM-1.1, no scattering peak can be observed, indicating a completely disordered structure. Interestingly, the as-obtained CM-1.3 has two evident scattering peaks, indicating the formation of an ordered mesoporous structure. However, the 20 scattering peak shifts to a low $q$-value, indicating framework expansion. Furthermore, the scattering peaks of CM-1.5 gradually become more intensely with the increase in $\mathrm{EO} / \mathrm{PO}$, indicating the formation of a highly ordered mesoporous structure. Meantime, the 10 and 20 scattering peaks shift to a high $q$-value, indicating framework shrinkage. These results display the effect of the $\mathrm{EO} / \mathrm{PO}$ ratio on the formation of porous structures transforming from ordered, to disordered, and then to ordered porous structures. 


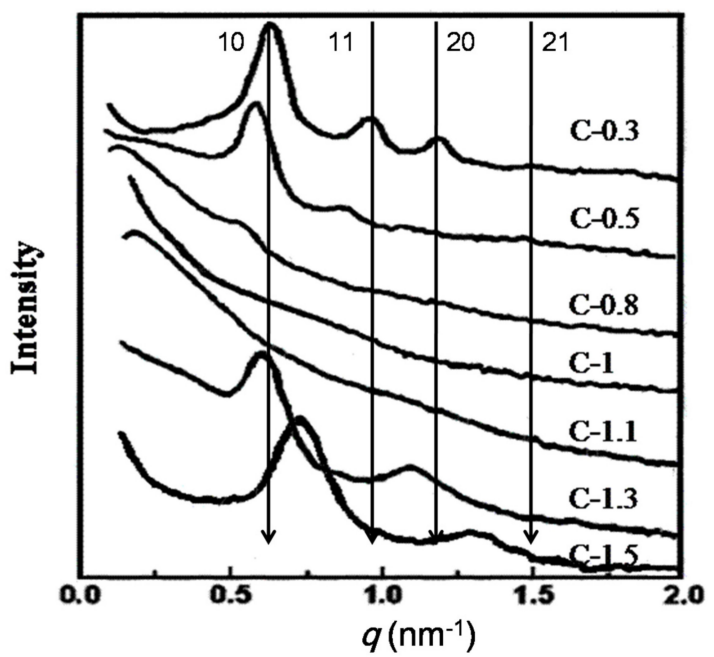

Figure 1. Small-angle X-ray scattering spectra of carbons prepared at different conditions.

TEM images of mesoporous carbons are showed in Figure 2. It can be seen that the EO/PO ratio has a significant effect on the formation of the mesoporous structures of carbons. The C- 0.3 sample possesses vortex-like mesopores, while the amount of vortex-like mesopores reduces and the size of vortex-like mesopores becomes large in the C- 0.5 sample. Obviously, with the increase in EO/PO ratio, the porous structure gradually becomes disordered. The C- 0.8 sample exhibits a typical disordered mesoporous structure with a few vortex-like pores, and the C-1 sample exhibits a worm-like porous structure. However, ordered porous structures of carbons are gradually formed with the further increase in $\mathrm{EO} / \mathrm{PO}$ ratio. The stripe-like structure appears when $\mathrm{EO} / \mathrm{PO}$ reaches 1.1. The $\mathrm{C}-1.3$ sample prepared with $\mathrm{EO} / \mathrm{PO}=1.3$ shows an ordered hexagonal porous structure. Furthermore, a well ordered two-dimensional hexagonal porous structure is formed when $\mathrm{EO} / \mathrm{PO}$ reaches 1.5, which is consistent with the result of SAXS analysis. The effect of the EO/PO ratio on the formation of porous structures mainly depends on the different interfacial curvature [26]. The formation of well-ordered vortex-like mesopores may be due to the fact that P123 with fewer EO units has high contraction in the axial direction, weakening the interfacial curvature. As the $\mathrm{EO} / \mathrm{PO}$ ratio increases, the hydrophilic groups gradually increase, leading to the enhancement of interface curvature that can assemble into the ordered stripe-like mesoporous structure. The weaker interfacial curvature prefers to aggregate into vortex-like microdomains, while the higher interfacial curvature prefers to aggregate into stripe-like microdomains [27].
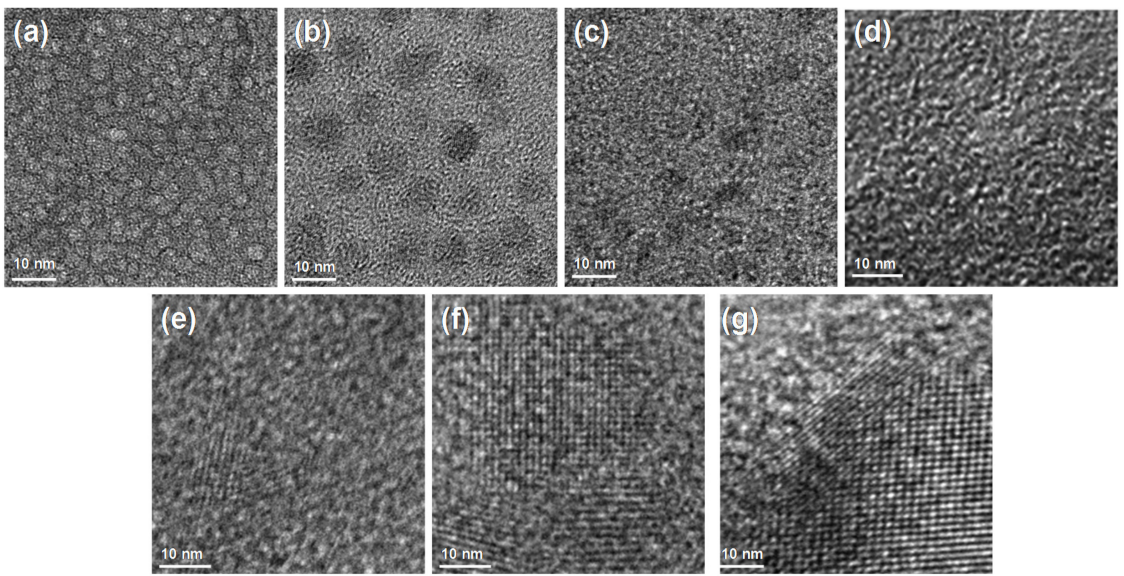

Figure 2. TEM images of carbons prepared at different conditions: (a) C-0.3; (b) C-0.5; (c) C-0.8; (d) C-1; (e) C-1.1; (f) C-1.3; (g) C-1.5. 
The $N_{2}$ sorption isotherms and the corresponding pore size distribution curves of mesoporous carbons are displayed in Figure 3. The all samples exhibit a significant $N_{2}$ adsorption at the relative pressure $\left(P / P_{0}\right)$ below 0.1 , indicating the formation of abundant micropores. As shown in Figure 3a, the amount of the $N_{2}$ adsorption at $P / P_{0}=0.1$ gradually become larger with the increase in $\mathrm{EO} / \mathrm{PO}$ ratio from 0.3 to 1 . Meanwhile, a hysteresis loop in a $P / P_{0}$ range of $0.45-1.0$ is observed, indicating a typical characteristic of mesoporous structure. The hysteresis loop of $\mathrm{C}-1$ can be categorized into the $\mathrm{H}_{3}$-type isotherm according to the IUPAC classification, which indicates that the $\mathrm{C}-1$ sample possesses slit pores and some large pores. The C-0.8 sample shows a slight change in the hysteresis loop and a distinct decrease in nitrogen sorption at a relative pressure $P / P_{0}$ below 0.1 , indicating the main decrease in micropores. However, the hysteresis loops of C- 0.5 and C- 0.3 have a distinguishing change, which can be classified as an $\mathrm{H}_{1}$-type isotherm, indicating the formation of vortex-like pores with a narrow pore size distribution. Furthermore, the $\mathrm{H}_{1}$-type hysteresis loop of the $\mathrm{C}-0.3$ sample increases in the $P / P_{0}$ range of $0.42-1.0$, and a slight decrease in nitrogen sorption at a relative pressure $P / P_{0}$ below 0.1 shows the increase in mesopores. Compared with the $C-1$ sample (Figure $3 b$ ), the $C-1.1$ sample shows a slightly decrease in nitrogen sorption at a relative pressure $P / P_{0}$ of $0.1-0.45$, and the $\mathrm{H}_{1}$-type hysteresis loop slightly changes. However, the $\mathrm{H}_{3}$-type hysteresis loop of the $\mathrm{C}-1.3$ sample distinctly reduces, indicating the decrease in mesopores. Moreover, the C-1.5 sample exhibits a small $\mathrm{H}_{1}$-type hysteresis loop, indicating the formation of mesoporous carbons. These results demonstrate that the $\mathrm{EO} / \mathrm{PO}$ ratio is the key issue on the formation of porous structure. The carbons prepared with $\mathrm{EO} / \mathrm{PO}=1$ shows the highest adsorption. This is probably due to the synergistic effect of copolymers forming abundant porous structures. The mixture of $\mathrm{EO}$ and PO blocks can affect the formation of interconnected microtunnels between the larch resins and the copolymers [28]. The pore size distribution curves of carbons are shown in Figure 3c,d. The C-0.3 sample possesses the maxima centered at 1.2, 3.5, and $6.4 \mathrm{~nm}$. The pores of C-1 sample become wider and centered at 1.3, 3.4, and $17.2 \mathrm{~nm}$ with the broadest pore size distribution. This is because of the collapse of pores caused by the synergistic effect of EO and PO groups after carbonization. The maxima of carbons prepared with high $\mathrm{EO} / \mathrm{PO}$ ratio also gradually shifts to a narrow pore, and C-1.5 has maxima centered at 1.2 and $3.4 \mathrm{~nm}$, which is relative to the amount of PO groups. These results indicate that the EO/PO ratio has a significant effect on the formation of porous structures.
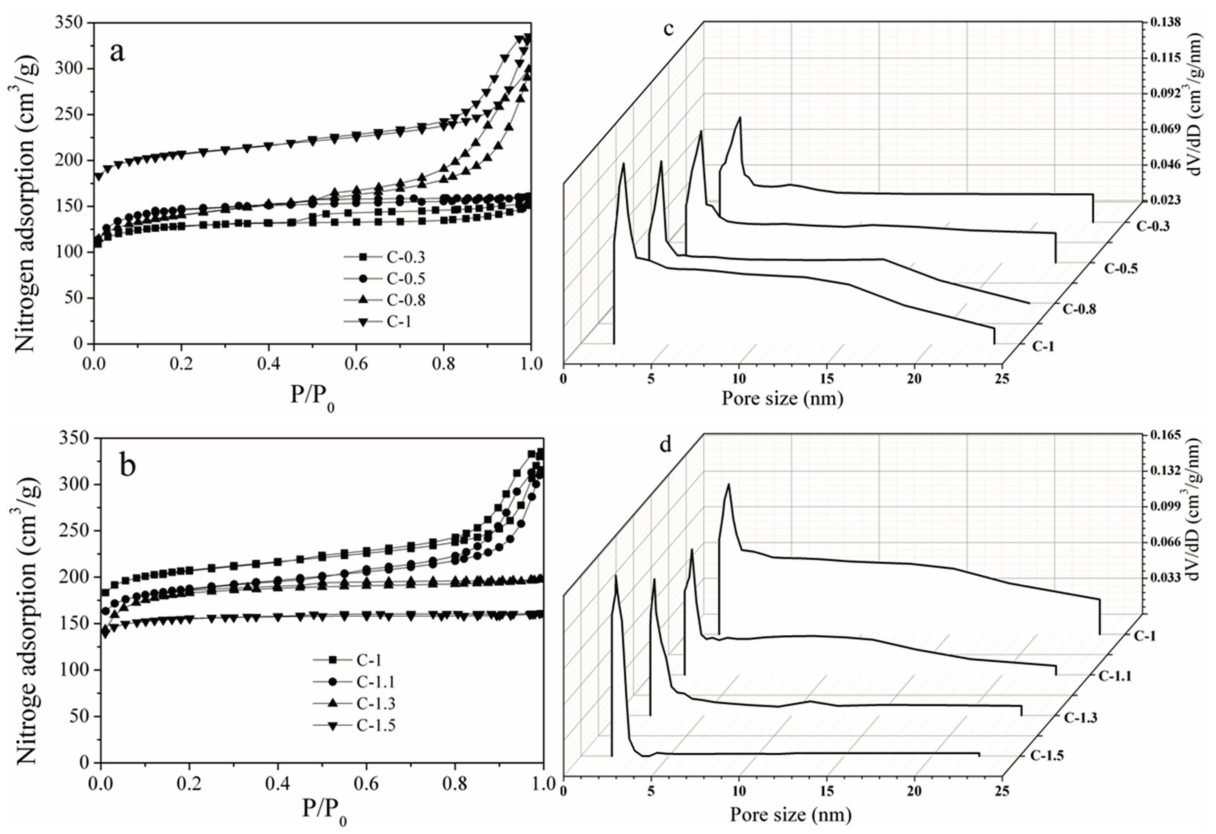

Figure 3. Nitrogen sorption isotherms $(\mathbf{a}, \mathbf{b})$ and pore size distribution curves $(\mathbf{c}, \mathbf{d})$ of carbons prepared at different conditions. 
The textual parameters of the C-y materials are presented in Table 1. The C-1 sample possesses the highest $S_{\text {BET }}$ (up to $634 \mathrm{~m}^{2} \cdot \mathrm{g}^{-1}$ ), and the $S_{\text {meso }} / S_{\text {BET }}$ of $C-1$ reach $20 \%$. This is due to the strong synergistic effect of equaled $\mathrm{EO}$ and $\mathrm{PO}$ units. As the $\mathrm{EO} / \mathrm{PO}$ ratio decreases from 1 to 0.3 , the $\mathrm{S}_{\mathrm{BET}}$ of carbons decreases from 637 to $398 \mathrm{~m}^{2} \cdot \mathrm{g}^{-1}$, and the $\mathrm{S}_{\text {meso }} / \mathrm{S}_{\text {BET }}$ ratio decreases from $20 \%$ to $14 \%$. The $\mathrm{S}_{\text {BET }}$ of carbons decreases from 637 to $475 \mathrm{~m}^{2} \cdot \mathrm{g}^{-1}$, and the $\mathrm{S}_{\text {meso }} / \mathrm{S}_{\text {BET }}$ decreases from $20 \%$ to $12 \%$ when the EO/PO increases from 1 to 1.5, respectively. These results indicate that the EO/PO ratio is of great importance in controlling the porous structure.

Table 1. Textual parameters of carbons prepared at different conditions.

\begin{tabular}{|c|c|c|c|}
\hline Sample & $S_{\text {BET }}\left(\mathrm{m}^{2} / \mathrm{g}\right)$ & $\mathrm{S}_{\text {meso }} / \mathrm{S}_{\text {BET }}(\%)$ & $\mathrm{S}_{\text {micro }} / \mathrm{S}_{\text {BET }}(\%)$ \\
\hline C-0.3 & 393 & 14 & 86 \\
\hline C- 0.5 & 410 & 17 & 83 \\
\hline C-0.8 & 421 & 20 & 80 \\
\hline C-1 & 634 & 20 & 80 \\
\hline C-1.1 & 601 & 18 & 82 \\
\hline C-1.3 & 569 & 14 & 86 \\
\hline C-1.5 & 475 & 12 & 88 \\
\hline
\end{tabular}

\subsection{Formation Mechanism of Mesoporous Carbons}

Mesoporous carbons with different porous structures have been synthesized via a facile method, and the formation of the mesostructure is related to the co-operative self-assemble mechanism, as shown in Figure 4. We prepared the liquefied larch via phenol liquefication, which has a condensation reaction with formaldehyde to form larch-based resins under basic conditions. The obtained resin precursors with abundant hydroxyl can further interact with EO units via hydrogen bond forming resin-copolymers composites, and the copolymer micelles penetrate into the EO-PO interface, which assembles in large voids to form mesopores with various ordered mesostructure symmetries [29]. After carbonization at $700{ }^{\circ} \mathrm{C}$, soft templates are removed and the corresponding mesopores are opened. Additionally, the hydrophilic and hydrophobic segments have a strong interaction with each other, which can affect the porous structure of carbons. When the EO units are equal to the PO units, the composites have a strong interaction due to their synergistic effect [30], resulting in the collapse of the mesostructure after carbonization. The stripe-like micelles are formed when the ratio of $\mathrm{EO} / \mathrm{PO}$ is bigger than 1 , which is due to the strengthening ability of the hydrophilic segment with high interface curvature. The high interface curvature can maintain the stability of the stripe-like micelles and form a rigid framework after carbonization. On the other hand, the vortex-like micelles are formed when the ratio of $\mathrm{EO} / \mathrm{PO}$ is smaller than 1, which is ascribed to the strengthening ability of the hydrophobic segment surrounding the hydrophilic segments with low interface curvature. The low interface curvature can maintain the stable spherical micelles and form rigid framework after carbonization. 


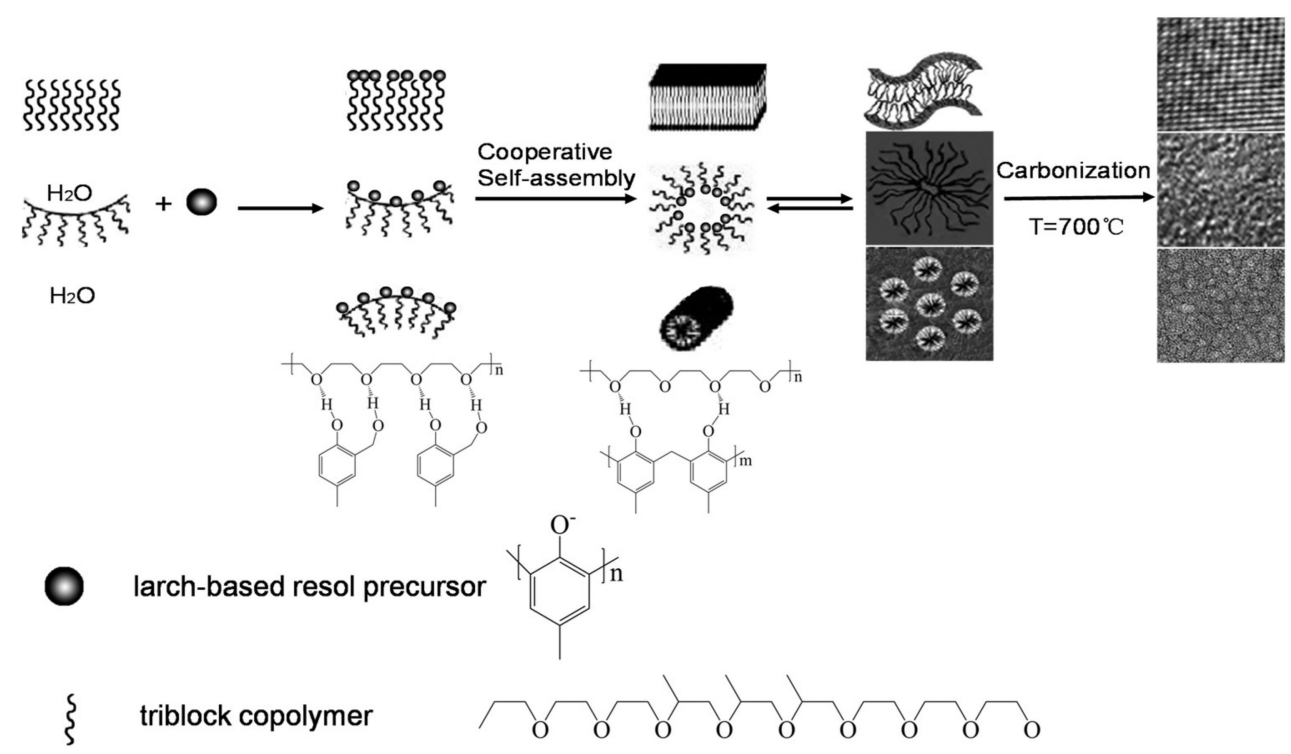

Figure 4. Schematic illustration of the proposed mechanism for the formation of the mesoporous carbons.

\subsection{Electrochemical Properties}

The mesoporous carbons with well-developed porosity were also evaluated for electrode materials in supercapacitors. The cyclic voltammetry and galvanostatic charge/discharge tests were employed to characterize the capacitive properties. Figure 5 a shows the cyclic voltammetry curves of C-0.3 at scan rates from 1 to $200 \mathrm{mV} \cdot \mathrm{s}^{-1}$. The cyclic voltammetry curves measured at low scan rates show a nearly rectangular shape, suggesting a double-layer capacitance behavior. However, the shape significantly changes from a rectangular shape to polar curves with the increase in scan rate, indicating the low conductivity of carbons. The charge-discharge plots of C- 0.3 measured at current densities from 0.1 to $1 \mathrm{~A} \cdot \mathrm{g}^{-1}$ are shown in Figure $5 \mathrm{~b}$. The charge-discharge curves have a distinct arc with a small IR drop due to the low conductivity of the electrode materials. The specific capacitance of C-0.3 is calculated using discharge plots, which is $79 \mathrm{~F} \cdot \mathrm{g}^{-1}$ at a current density of $1 \mathrm{~A} \cdot \mathrm{g}^{-1}$. This indicates that C-0.3 possesses a poor capacitive performance.
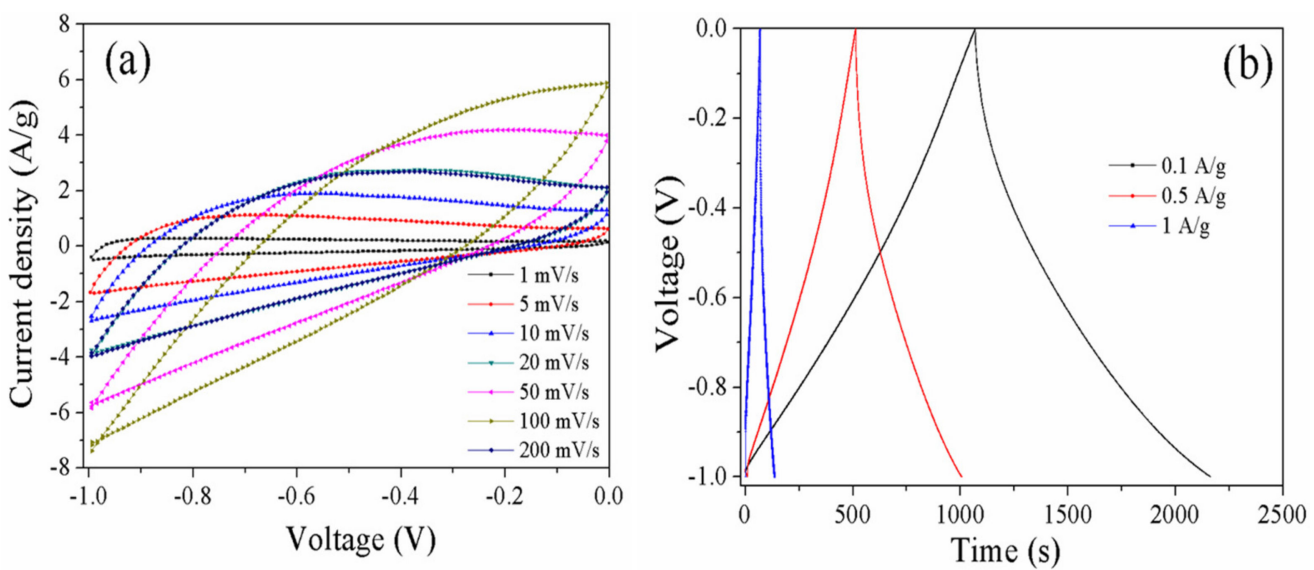

Figure 5. CV curves of C-0.3 at different scan rates varying from 1 to $200 \mathrm{mV} \cdot \mathrm{s}^{-1}$ (a) and charge-discharge curves at different current densities from 0.1 to $1 \mathrm{~A} \cdot \mathrm{g}^{-1}(\mathbf{b})$.

As can be seen in Figure 6a, the CV curves of C-1 show a nearly rectangular shape at the low scan rates, suggesting a double-layer capacitance behavior. However, the shape slightly changes from a rectangular shape to a polar curve with the increase in scan rate, indicating the low conductivity of 
carbons. The charge-discharge plots of C-1 measured at current densities from 0.2 to $10 \mathrm{~A} \cdot \mathrm{g}^{-1}$ are shown in Figure $6 \mathrm{~b}$. The charge-discharge curves have a distinct arc with a small IR drop due to the low electronic conductivity of the electrode materials. The specific capacitance of C-1 measured at a current density of $1 \mathrm{~A} \cdot \mathrm{g}^{-1}$ is $158 \mathrm{~F} \cdot \mathrm{g}^{-1}$, which is better than that of $\mathrm{C}-0.3$. However, the specific capacitance only retains $103 \mathrm{~F} \cdot \mathrm{g}^{-1}$ when the current density increases to $10 \mathrm{~A} \cdot \mathrm{g}^{-1}$. This indicates that C-1 possess a moderate capacitive performance and poor electronic conductivity.
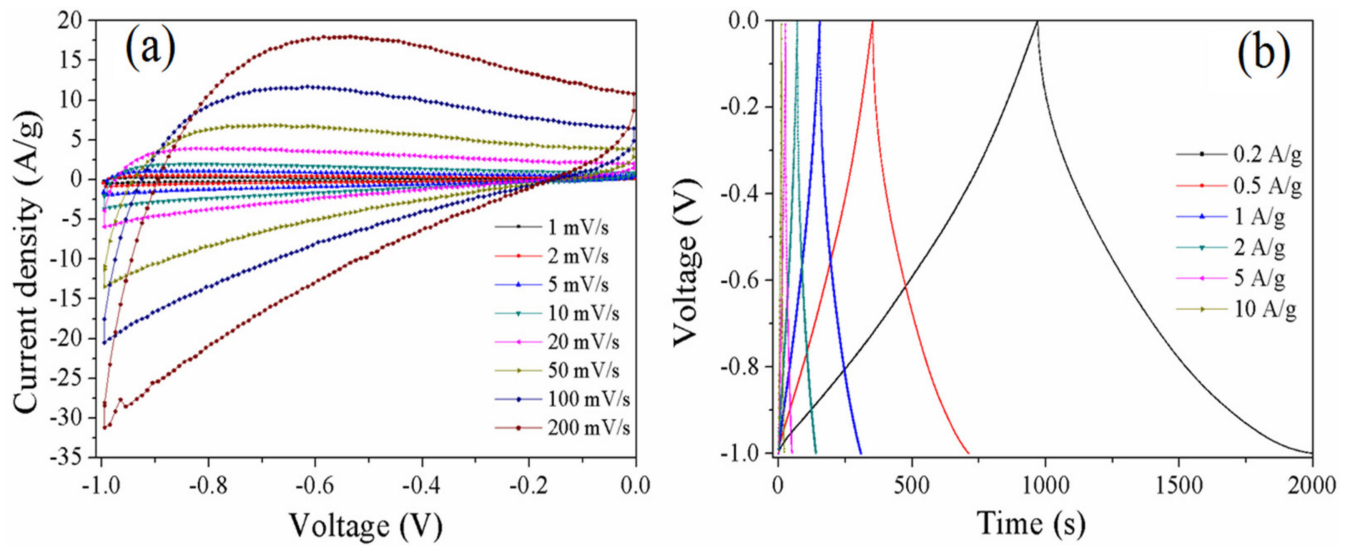

Figure 6. CV curves of C-1 at different scan rates varying from 1 to $200 \mathrm{mV} \cdot \mathrm{s}^{-1}$ (a) and charge-discharge curves at different current densities from 0.2 to $10 \mathrm{~A} \cdot \mathrm{g}^{-1}$ (b).

The CV curves of C-1.5 showed in Figure 7a possess a nearly rectangular shape in the low scan rates, indicating a double-layer capacitance behavior. Furthermore, the shape has no apparent change with the increase in scan rate, indicating excellent conductivity due to the ordered mesoporous structure. The charge-discharge plots of C-1.5 measured at current densities from 0.2 to $10 \mathrm{~A} \cdot \mathrm{g}^{-1}$ are shown in Figure $7 \mathrm{~b}$. The charge-discharge curves show isosceles triangles due to the excellent conductivity of the electrode materials. The specific capacitance of C-1.5 measured at a current density of $1 \mathrm{~A} \cdot \mathrm{g}^{-1}$ is $125 \mathrm{~F} \cdot \mathrm{g}^{-1}$, and the specific capacitance retains $107 \mathrm{~F} \cdot \mathrm{g}^{-1}$ when the current density increases to $10 \mathrm{~A} \cdot \mathrm{g}^{-1}$, indicating superior conductivity due to the well-defined porosity. The capacitance drop of all the carbons at high current density can be illustrated by the assumption that the charge diffusion in the pores is interrupted owing to the time prevent to rate of charge/discharge [31].
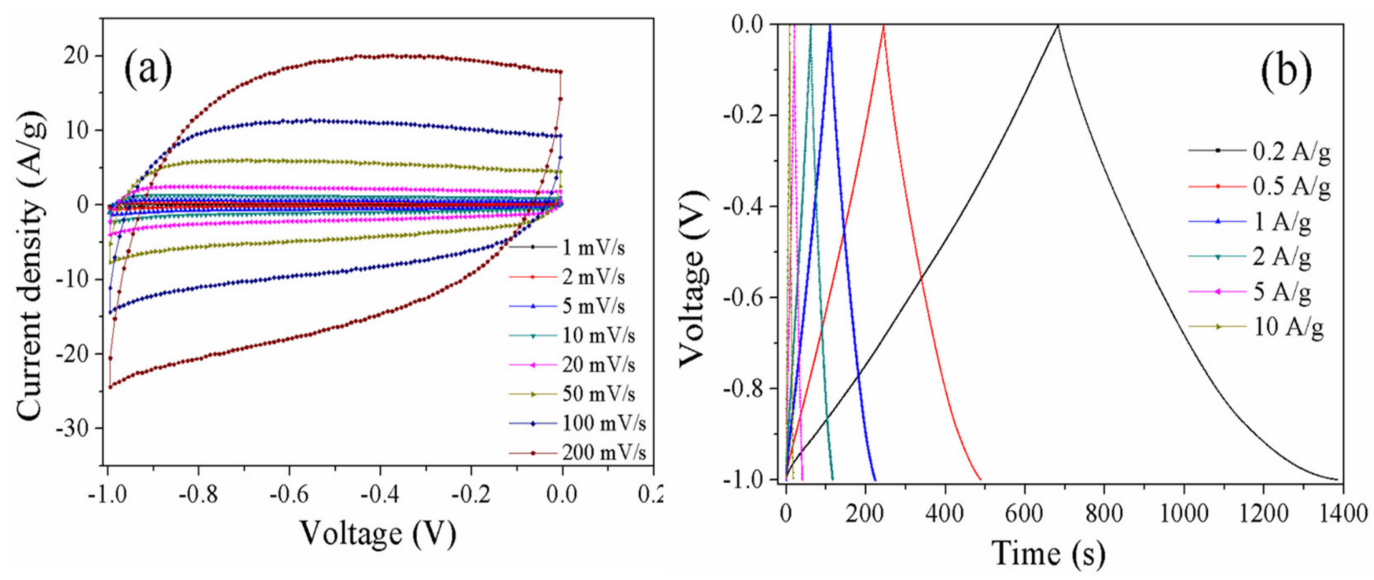

Figure 7. CV curves of C-1.5 at different scan rates varying from 1 to $200 \mathrm{mV} \cdot \mathrm{s}^{-1}$ (a) and charge-discharge curves at different current densities from 0.2 to $10 \mathrm{~A} \cdot \mathrm{g}^{-1}$ (b).

To further investigate the electrochemical performance of the carbons, the circulations and EIS were systematically evaluated. The cycling stability tests of the carbons were performed at a current 
density of $10 \mathrm{~A} \cdot \mathrm{g}^{-1}$ for 2000 galvanostatic charge/discharge cycles, as shown in Figure 8a. It can be seen that the specific capacitances of the carbons decrease slightly and remain above $98 \%$ of the maximum capacitance after 2000 cycles, indicating that the electrode has good electrochemical stability and a high degree of reversibility due to the presence of the meropores. Electrochemical impedance spectroscopy (EIS) analysis was used to gain a deep insight into the resistive and capacitive behavior of the carbons. As shown in Figure $8 b$, the slope of the curves shows an electric double-layer capacitive behavior at low frequencies. However, C-1.5 has the highest slope, indicating the lowest resistance. In the region of medium frequencies, the Warburg impedance of all samples is observed, where the electrolyte ion penetrate into the depth of the micro-/mesopores of the electrode [32]. At high frequencies, the capacitive behavior in EIS is illustrated by the semi-circle plots, a small semicircle indicates a low contact resistance, which is related to the interaction between porous structure of the electrode and ions [33]. This is related to the interaction of the porous structure of the electrode and ions. C- 0.3 and C-1.5, compared to C-1, have smaller radii due to the presence of order mesopores. At high frequencies, the capacitive performance is poor because it is difficult to access the rapid variation of the potential by the transfer of abundant ions [34]. In the region of medium frequency, the Warburg impedance of samples were observed, where the electrolyte ions penetrate into the depth of the pores of the electrode. At a low frequency, a straight line demonstrates the ideal electric double-layer capacitive behavior of electrode materials [35]. The low internal resistance has a positive effect on conductivity. These results correspond to the analysis of CV and GCD curves. Consequently, C-1.5 has well-developed porosity and a high surface area, resulting in low resistance, which exhibits excellent electrochemical performance and shows potential for industrial applications.
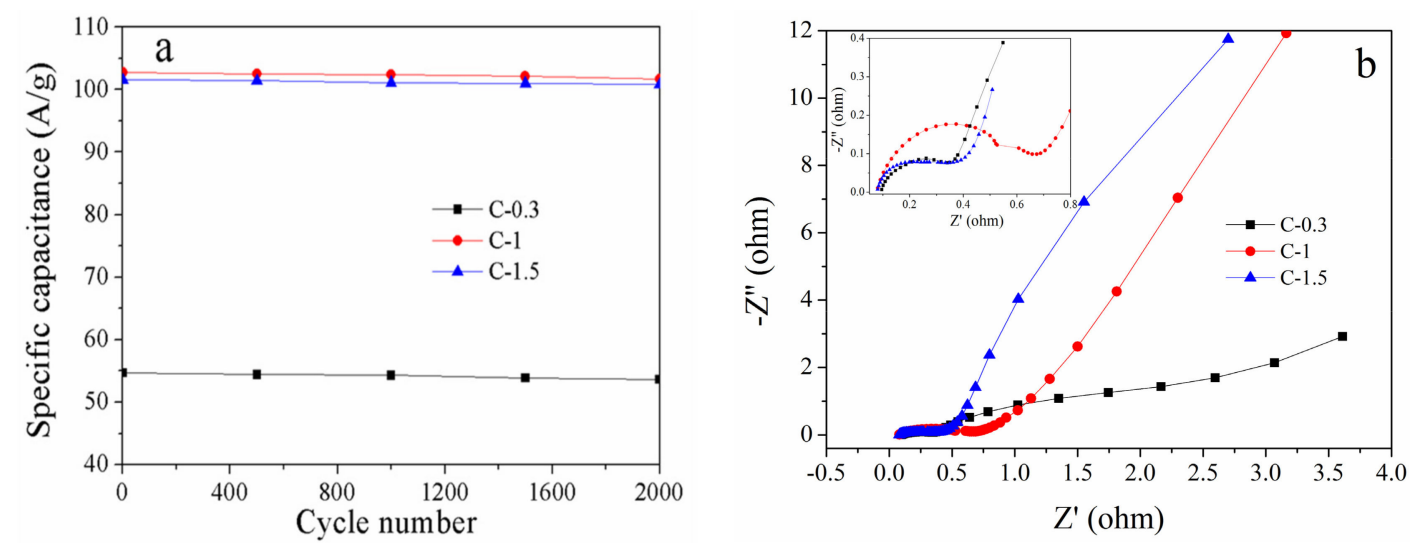

Figure 8. The cycling stability measured at $10 \mathrm{~A} \cdot \mathrm{g}^{-1}$ (a) and Nyquist plots of impedance spectra (b) of the mesoporous carbons.

\section{Conclusions}

We report a facile and reproducible approach for the synthesis of mesoporous carbons with different porous structures and tunable pore sizes by using a mixture of F127 and P123 as templates and larch-based resins as carbon sources. The change in porous structure from worm-like to 2D hexagonal structure was observed with the increase in $\mathrm{EO} / \mathrm{PO}$ ratio from 1 to 1.5. However, the porous structure changes from a worm-like to a vortex-like porous structure when EO/PO ratio decreases from 1 to 0.3 . The mesoporous carbons where $\mathrm{EO} / \mathrm{PO}=1$ have the highest surface area. Meanwhile, the pore size of all carbons is mainly distributed at $1.8-3.0 \mathrm{~nm}$. The $S_{\text {BET }}$ of carbons decreases from 634 to $398 \mathrm{~m}^{2} \cdot \mathrm{g}^{-1}$ as the EO/PO ratio decreases from 1 to 0.3 . The $S_{\mathrm{BET}}$ of carbons decreases from 634 to $475 \mathrm{~m}^{2} \cdot \mathrm{g}^{-1}$ when the $\mathrm{EO} / \mathrm{PO}$ increases from 1 to 1.5. Indeed, the pore size depends on the length of the hydrophobic PO blocks in the mixture of F127 and P123. The probable cooperative assembly mechanism of mesoporous carbons is proposed for the synthesis route. Furthermore, the electrochemical experimental results demonstrate that the mesoporous carbons exhibit high specific capacitance, superior electrochemical 
stability, and a high degree of reversibility. In addition, the carbon where $\mathrm{EO} / \mathrm{PO}=1.5$ has a high specific capacitance of $107 \mathrm{~F} \cdot \mathrm{g}^{-1}$ in $6 \mathrm{M} \mathrm{KOH}$ at a current density of $10 \mathrm{~A} \cdot \mathrm{g}^{-1}$, and the carbon where $\mathrm{EO} / \mathrm{PO}=1.5$ exhibits excellent conductivity, which is suitable for industrial applications.

Acknowledgments: The present work was financially supported by the National Key Technology R\&D Program (2015BAD14B06), the Natural Science Foundation of Shandong (ZR2017LEM009), and the National Natural Science Foundation of China (Grant No. 31500489, 31600472, 31570566, 31570567).

Author Contributions: Shouxin Liu and Xin Zhao conceived and designed the experiments; Wei Li performed the experiments; Honglei Chen and Shoujuan analyzed the data; Shouxin Liu and Fangong Kong contributed reagents/materials/analysis tools; Xin Zhao wrote the paper.

Conflicts of Interest: The authors declare no conflict of interest.

\section{References}

1. Li, Z.C.; Zhai, K.; Wang, G.; Li, Q.; Guo, P. Preparation and Electrocapacitive Properties of Hierarchical Porous Carbons Based on Loofah Sponge. Materials 2016, 9, 912. [CrossRef] [PubMed]

2. He, X.J.; Ma, H.; Wang, J.X.; Xie, Y.Y. Porous carbon nanosheets from coal tar from high-performance supercapacitors. J. Power Sources 2017, 357, 41-46. [CrossRef]

3. Wang, Z.Q.; Sun, L.X.; Xu, F.; Zou, Y.J.; Chu, H.L.; Zhu, M. Synthesis of N-doped hierarchical carbon spheres for $\mathrm{CO}_{2}$ capture and supercapacitors. RSC Adv. 2016, 6, 1422-1427. [CrossRef]

4. Fletcher, S.; Kirkpatrick, I.; Dring, R.; Puttock, R.; Howroyd, S. The modelling of carbon-based supercapacitors: Distributions of time constants and Pascal Equivalent Circuits. J. Power Sources 2017, 345, 247-253. [CrossRef]

5. Zu, G.; Shen, J.; Zou, L.; Wang, F.; Wang, X.; Yao, X. Nanocellulose-derived highly porous carbon aerogels for supercapacitors. Carbon 2016, 99, 203-211. [CrossRef]

6. Borchardt, L.; Oschatz, M.; Kaskel, S. Tailoring porosity in carbon materials for supercapacitor applications. Mater. Horiz. 2014, 1, 157-168. [CrossRef]

7. Zhuo, H.; Hu, Y.J.; Tong, X.; Zhong, L.X.; Sun, R.C. Sustainable hierarchical porous carbon aerogel from cellulose for high-performance supercapacitor and $\mathrm{CO}_{2}$ capture. Ind. Crops Prod. 2016, 87, 229-235. [CrossRef]

8. Fuertes, A.B.; Sevilla, M. High-surface area carbons from renewable sources with a bimodal micro-mesoporosity for high-performance ionic liquid-based supercapacitors. Carbon 2015, 94, 41-52. [CrossRef]

9. Li, W.Q.; Liu, S.; Pan, N.; Zeng, F.j.; Liang, Y. Post-treatment-free synthesis of highly mesoporous carbon for high-performance supercapacitor in aqueous electrolytes. J. Power Sources 2017, 357, 138-143. [CrossRef]

10. Ahmed, A.; Surjith, A.; Kateryna, B.; Mohan, V.J. Review on the Antimicrobial Properties of Carbon Nanostructures. Materials 2017, 10, 1066.

11. Bhattacharjya, D.; Kim, M.S.; Yu, J.S. High performance supercapacitor prepared from hollow mesoporous carbon capsules with hierarchical nanoarchitecture. J. Power Sources 2013, 244, 799-805. [CrossRef]

12. Ma, X.; Gan, L.; Liu, M.; Tripathi, P.K.; Chen, L. Mesoporous size controllable carbon microspheres and their electrochemical performances for supercapacitor electrodes. J. Mater. Chem. A 2014, 2, 8407-8415. [CrossRef]

13. Zhao, Y.; Zhang, X.M.; He, Y.; Liu, N.; Tan, T.; Liang, C. Biomass Derived Nitrogen-Doped Highly Porous Carbon Material with a Hierarchical Porous Structure for High-Performance Lithium/Sulfur Batteries. Materials 2017, 10, 1158. [CrossRef] [PubMed]

14. Lv, Y.; Zhang, F.; Dou, Y.; Tu, B.; Zhao, D. A comprehensive study on $\mathrm{KOH}$ activation of ordered mesoporous carbons and their supercapacitor application. J. Mater. Chem. 2012, 22, 93-99. [CrossRef]

15. Ma, T.Y.; Liu, L.; Yuan, Z.Y. Direct synthesis of ordered mesoporous carbons. Chem. Soc. Rev. 2013, 42, 3977-4003. [CrossRef] [PubMed]

16. Xia, Y.D.; Yang, Z.X.; Mokaya, R. Templated nanoscale porous carbons. Nanoscale 2010, 2, 639-659. [CrossRef] [PubMed]

17. Chuenchom, L.; Kraehnert, R.; Smarsly, B.M. Recent progress in soft-templating of porous carbon materials. Soft Matter 2012, 8, 10801-10812. [CrossRef]

18. Braghiroli, F.L.; Fierro, V.; Parmentier, J.; Celzard, A. Easy and eco-friendly synthesis of ordered mesoporous carbons by self-assembly of tannin with a block copolymer. Green Chem. 2016, 18, 3265-3271. [CrossRef] 
19. Wang, C.J.; Wu, D.P.; Wang, H.J.; Gao, Z.Y.; Jiang, K. Nitrogen-doped two-dimensional porous carbon sheets derived from clover biomass for high-performance supercapacitors. J. Power Sources 2017, 363, 375-383. [CrossRef]

20. Nelson, K.M.; Mahurin, S.M.; Mayes, R.T.; Teague, C.M.; Dai, S. Preparation and $\mathrm{CO}_{2}$ adsorption properties of soft-templated mesoporous carbons derived from chestnut tannin precursors. Microporous Mesoporous Mater. 2016, 222, 94-103. [CrossRef]

21. Xu, F.; Chen, Y.; Tang, M.; Wang, H.; Deng, J.; Wang, Y. Acid induced self-assembly strategy to synthesize ordered mesoporous carbons from biomass. ACS Sustain. Chem. Eng. 2016, 4, 4473-4479. [CrossRef]

22. Li, W.; Huang, Z.; Wu, Y.; Zhao, X.; Liu, S. Honeycomb carbon foams with tunable pore structures prepared from liquefied larch sawdust by self-foaming. Ind. Crops Prod. 2015, 64, 215-223. [CrossRef]

23. Zhao, X.; Li, W.; Liu, S.X. Coupled soft-template/hydrothermal process synthesis of mesoporous carbon spheres from liquefied larch sawdust. Mater. Lett. 2013, 107, 5-8. [CrossRef]

24. Zhao, X.; Li, W.; Zhang, S.; Liu, L.; Liu, S.X. Facile fabrication of hollow and honeycomb-like carbon spheres from liquefied larch sawdust via ultrasonic spray pyrolysis. Mater. Lett. 2015, 157, 135-138. [CrossRef]

25. Zhao, X.; Li, W.; Liu, S. Ordered mesoporous carbon membrane prepared from liquefied larch by a soft method. Mater. Lett. 2014, 126, 174-177. [CrossRef]

26. Meng, Y.; Gu, D.; Zhang, F.Q.; Wan, Y.; Stein, A.; Zhao, D.Y. A family of highly ordered mesoporous polymer resin and carbon structures from organic-organic self-assembly. Chem. Mater. 2006, 18, 4447-4464. [CrossRef]

27. Zhang, F.Q.; Meng, Y.; Gu, D.; Yan, Y.; Zhao, D.Y. An aqueous cooperative assembly route to synthesize ordered mesoporous carbons with controlled structures and morphology. Chem. Mater. 2006, 18, 5279-5288. [CrossRef]

28. Mai, Y.; Eisenberg, A. Self-assembly of block copolymers. Chem. Soc. Rev. 2012, 41, 5969-5985. [CrossRef] [PubMed]

29. Wang, R.; Li, W.; Liu, S.X. A porous carbon foam prepared from liquefied birch sawdust. J. Mater. Sci. 2012, 47, 1977-1984. [CrossRef]

30. Jin, J.; Nishiyama, N.; Egashira, Y.; Ueyama, K. Pore structure and pore size controls of ordered mesoporous carbons prepared from resorcinol/formaldehyde/triblock polymers. Microporous Mesoporous Mater. 2009, 118, 218-223. [CrossRef]

31. Hao, L.; Li, X.; Zhi, L. Carbonaceous electrode materials for supercapacitors. Adv. Mater. 2013, 25, 3899-3904. [CrossRef] [PubMed]

32. Wang, H.; Li, Z.; Tak, J.K.; Holt, C.M.B.; Stephenson, T.; Mitlin, D. Supercapacitors based on carbons with tuned porosity derived from paper pulp mill sludge biowaste. Carbon 2013, 57, 317-328. [CrossRef]

33. Wang, Q.; Yan, J.; Wang, Y.; Wei, T.; Jing, X. Three-dimensional flower-like and hierarchical porous carbon materials as high-rate performance electrodes for supercapacitors. Carbon 2014, 67, 119-127. [CrossRef]

34. Sevilla, M.; Mokaya, R. Energy storage applications of activated carbons: Supercapacitors and hydrogen storage. Energy Environ. Sci. 2014, 7, 1250. [CrossRef]

35. Zhang, W.; Lin, H.; Lin, Z.; Yin, J.; Lu, H.; Zhao, M. 3D hierarchical porous carbon for supercapacitors prepared from lignin through a facile template-free method. ChemSusChem 2015, 8, 2114-2122. [CrossRef] [PubMed]

(C) 2017 by the authors. Licensee MDPI, Basel, Switzerland. This article is an open access article distributed under the terms and conditions of the Creative Commons Attribution (CC BY) license (http://creativecommons.org/licenses/by/4.0/). 